\title{
Antecubital Region
}

National Cancer Institute

\section{Source}

National Cancer Institute. Antecubital Region. NCI Thesaurus. Code C111032.

The area of the body that is anterior to the elbow. 